\title{
Tres-5: A Massive Jupiter-sized Planet Transiting a Cool G Dwarf
}

\section{Citation}

Mandushev, Georgi, Samuel N. Quinn, Lars A. Buchhave, Edward W. Dunham, Markus Rabus, Brian Oetiker, David W. Latham, et al. 2011. "TrES-5: A MASSIVE JUPITER-SIZED PLANET TRANSITING A COOL G DWARF." The Astrophysical Journal 741 (2): 114. https:// doi.org/10.1088/0004-637x/741/2/114.

\section{Permanent link}

http://nrs.harvard.edu/urn-3:HUL.InstRepos:41397478

\section{Terms of Use}

This article was downloaded from Harvard University's DASH repository, and is made available under the terms and conditions applicable to Other Posted Material, as set forth at http:// nrs.harvard.edu/urn-3:HUL.InstRepos:dash.current.terms-of-use\#LAA

\section{Share Your Story}

The Harvard community has made this article openly available. Please share how this access benefits you. Submit a story.

Accessibility 


\title{
TrES-5: A MASSIVE JUPITER-SIZED PLANET TRANSITING A COOL G DWARF
}

\author{
Georgi Mandushev $^{1}$, Samuel N. Quinn ${ }^{2}$, Lars A. Buchhave ${ }^{3}$, Edward W. Dunham ${ }^{1}$, Markus Rabus $^{4,5}$, Brian Oetiker $^{6}$, \\ David W. Latham ${ }^{2}$, David Charbonneau ${ }^{2}$, Timothy M. Brown ${ }^{7}$, Juan A. Belmonte ${ }^{5,8}$, and Francis T. O’ Donovan ${ }^{9}$ \\ ${ }^{1}$ Lowell Observatory, 1400 W Mars Hill Rd, Flagstaff, AZ 86001, USA; gmand@ lowell.edu \\ ${ }^{2}$ Harvard-Smithsonian Center for Astrophysics, 60 Garden St, Cambridge, MA 02138, USA \\ ${ }^{3}$ Niels Bohr Institute, Copenhagen University, DK-2100 Copenhagen, Denmark \\ ${ }^{4}$ Departamento de Astonomía y Astrofísica, Ponticia Universidad Católica de Chile, Casilla 306, Santiago 22, Chile \\ ${ }^{5}$ Instituto de Astrofísica de Canarias, C/ vía Láctea s/n, 38200 La Laguna, Tenerife, Spain \\ ${ }^{6}$ Department of Physics, Sam Houston State University, Huntsville, TX 77340, USA \\ ${ }^{7}$ Las Cumbres Observatory Global Telescope, 6740 Cortona Dr, Suite 102, Goleta, CA 93117, USA \\ ${ }^{8}$ Departamento de Astrofísica, Universidad de la Laguna, Tenerife, Spain \\ ${ }^{9}$ Ab Initio Software, 201 Spring St, Lexington, MA 02421, USA \\ Received 2011 August 11; accepted 2011 August 11; published 2011 October 25
}

\begin{abstract}
We report the discovery of TrES-5, a massive hot Jupiter that transits the star GSC 03949-00967 every 1.48 days. From spectroscopy of the star we estimate a stellar effective temperature of $T_{\text {eff }}=5171 \pm 36 \mathrm{~K}$, and from high-precision $B, R$, and $I$ photometry of the transit we constrain the ratio of the semimajor axis $a$ and the stellar radius $R_{\star}$ to be $a / R_{\star}=6.07 \pm 0.14$. We compare these values to model stellar isochrones to obtain a stellar mass of $M_{\star}=0.893 \pm 0.024 M_{\odot}$. Based on this estimate and the photometric time series, we constrain the stellar radius to be $R_{\star}=0.866 \pm 0.013 R_{\odot}$ and the planet radius to be $R_{\mathrm{p}}=1.209 \pm 0.021 R_{\mathrm{J}}$. We model our radial-velocity data assuming a circular orbit and find a planetary mass of $1.778 \pm 0.063 M_{\mathrm{J}}$. Our radial-velocity observations rule out line-bisector variations that would indicate a specious detection resulting from a blend of an eclipsing binary system. TrES-5 orbits one of the faintest stars with transiting planets found to date from the ground and demonstrates that precise photometry and followup spectroscopy are possible, albeit challenging, even for such faint stars.
\end{abstract}

Key words: planetary systems - techniques: photometric - techniques: radial velocities - techniques: spectroscopic

Online-only material: color figure

\section{INTRODUCTION}

Long before the initial discoveries of extrasolar planets around Sun-like stars by the Doppler technique (Latham et al. 1989; Mayor \& Queloz 1995), it was recognized that planets around other stars could also be detected photometrically if they transit their host stars (Struve 1952). Transit observations can provide at least two crucial pieces of information: the planet's size and orbital inclination. When combined with radialvelocity data they can be used to derive the planet's mass, surface gravity, and density, and infer its internal structure and composition. Many other studies also become possible for transiting planets around bright host stars, including analysis of the planet's atmosphere, temperature distribution, the presence of other bodies in the system, the spin-orbit alignment, etc. (see, e.g., Charbonneau et al. 2007).

The ever increasing number of discovered transiting planets, both from ground-based surveys and from the Kepler and CoRoT missions (Borucki et al. 2010; Baglin et al. 2009), shows the tremendous diversity of extrasolar planets and planetary systems. Particularly interesting are the relations between planetary mass, radius, and orbital period, as well as the distribution of planetary masses, as together they hold the key to understanding planetary formation and evolution. The current summary of transiting exoplanets at http://exoplanet.eu indicates that $70 \%$ of the transiting planets with known masses have masses $M_{\mathrm{p}} \lesssim 1.5 M_{\mathrm{J}}$. This apparent drop in the number of massive planets (noted also by Southworth et al. 2009) cannot be explained by major selection effects. Massive planets are also large, and thus easier to discover, unlike lower-mass planets, whose radius increases monotonically with mass for non-irradiated planets with cores (Fortney et al. 2007).

We report here the discovery of the Jupiter-sized massive transiting planet TrES-5. The planet orbits one of the faintest stars yet $(V=13.72)$ with a transiting planet found from groundbased wide-field surveys and is one of the very few planets found around stars fainter than $V=13.5$, including the space-based Kepler and CoRoT missions. TrES-5 increases the relatively small number of well-characterized massive transiting planets. Such planets play an important role in the study of planet formation, migration, and evolution (Chatterjee et al. 2008; Baraffe et al. 2010) and it has been suggested (Southworth et al. 2009) that the massive extrasolar planets form a different planet population from the lower-mass ones.

\section{PHOTOMETRY AND SPECTROSCOPY}

We monitored a $5.8 \times 5.8$ field in Cygnus with the Lowell Observatory Planet Search Survey Telescope (PSST; Dunham et al. 2004) and the STARE telescope on the Canary Islands in Spain, between UT 2007 July 15 and UT 2007 October 7. Both telescopes are part of TrES (the other two telescopes-Sleuth, at Palomar Observatory, and WATTS, in Texas, were not operational at that time). The same field was observed again in 2009 and 2010 by PSST alone. All images were reduced, and photometry and transit search carried out as described in Dunham et al. (2004). Altogether PSST observed 13 full and partial transit-like events of the star GSC 03949-00967. The object was at the very edge of the PSST field and because of the slightly different field size and scale the star was not observed by 


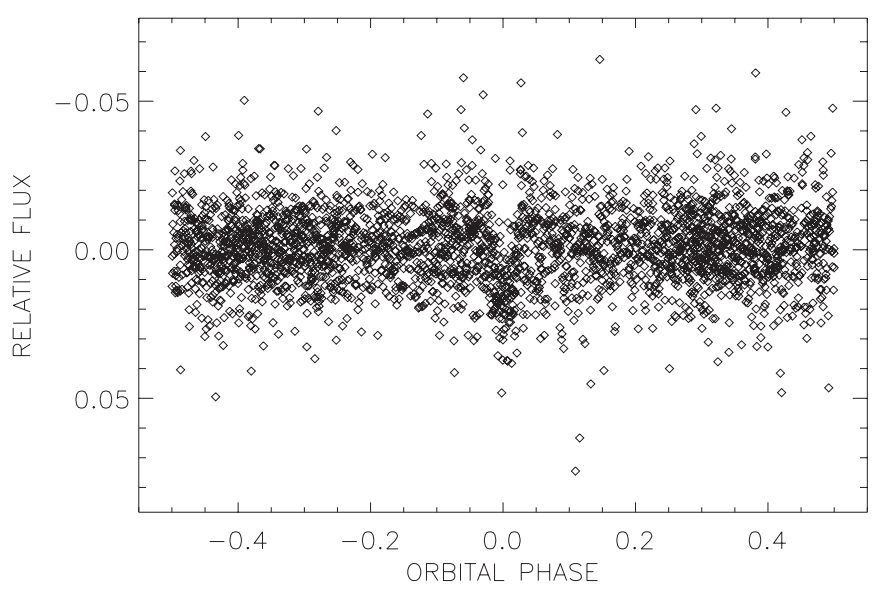

Figure 1. Discovery light curve of TrES-5. The plot shows the relative flux of the TrES-5 system as a function of the orbital phase, adopting the ephemeris in Table 3.

the STARE telescope. The depth and duration of the events were consistent with the transit of a Jupiter-sized planet across a $\mathrm{K}$ dwarf, and we undertook a program of follow-up observations to confirm the planetary nature of the object and measure its properties. The discovery light curve of TrES-5 is shown in Figure 1.

We carried out high-precision in-transit $B$-, $R$-, and $I$-band photometry of TrES-5 using Lowell Observatory's $0.8 \mathrm{~m}, 1.1 \mathrm{~m}$ Hall, and $1.8 \mathrm{~m}$ Perkins telescopes. We observed TrES-5 on UT 2009 November 17 with the $0.8 \mathrm{~m}$ telescope with NASACam in Cousins $R$ (47 exposures), on UT 2010 June 5 and 2010 November 30 with the $1.1 \mathrm{~m}$ Hall telescope with the NASA42 camera in Cousins $R$ and $I$ (87 and 108 exposures, respectively), and on UT 2010 September 5 with the $1.8 \mathrm{~m}$ Perkins telescope with PRISM in Johnson $B$ and Cousins $I$ (64 and 57 exposures, respectively). For all data sets, we derived differential fluxes relative to a large ensemble of local comparison stars. The highprecision Lowell Observatory photometry is shown in Figure 2.

In order to characterize the host star and provide an external check on the stellar parameters derived from the Tillinghast Reflector Echelle Spectrograph (TRES) spectra, we obtained off-transit $B V(R I)_{C}$ photometry of the TrES-5 system on UT 2010 October 10 and 11 with the $1.05 \mathrm{~m}$ Hall telescope at Lowell Observatory in combination with a $4 \mathrm{~K} \times 4 \mathrm{~K}$ e2 $\mathrm{v}$ CCD231 CCD detector. We calibrated the photometry using six standard fields from Landolt (1992). The results are listed in Table 1 together with other relevant data for the host star of TrES-5.

We observed the candidate with the TRES (Fürész 2008), mounted on the 60 inch Tillinghast Reflector at Fred L. Whipple Observatory on Mt. Hopkins in Arizona from 2010 September to 2011 April. We obtained 8 spectra, each consisting of 51 echelle orders and spanning the wavelength range 3850-9100 $\AA$, with a resolving power of $\lambda / \Delta \lambda \approx 44,000$. We obtained absolute radial velocities by cross-correlation against a synthetic template chosen from a large library of spectra based on Kurucz model atmospheres (see Nordström et al. 1994; Latham et al. 2002), calculated by John Laird and based on a line list compiled by Jon Morse. These velocities have a typical precision of $0.2 \mathrm{~km} \mathrm{~s}^{-1}$. However, the synthetic spectra only span a small portion of the total wavelength range, centered on the $\mathrm{Mg}$ b triplet at $5187 \AA$. We derived precise relative velocities by taking advantage of the full spectrum, cross-correlating all orders against those of an observed template. The radial velocity measurements and their errors are listed in Table 2. For a description of the reduction
Table 1

TrES-5 Host Star

\begin{tabular}{lccc}
\hline \hline Parameter & Units & Value & Source \\
\hline R.A. & $\mathrm{J} 2000.0$ & $20^{\mathrm{h}} 20^{\mathrm{m}} 53^{\mathrm{s}} .24$ & 1 \\
Decl. & $\mathrm{J} 2000.0$ & $+59^{\circ} 26^{\prime} 55^{\prime \prime} .6$ & 1 \\
$\mathrm{GSC}$ & & $03949-00967$ & \\
{$\left[\mu_{\alpha}, \mu_{\delta}\right]$} & mas yr $^{-1}$ & {$[+10.4,+30.5]$} & 1 \\
$V$ & & $13.718 \pm 0.005$ & 2 \\
$B-V$ & & $0.927 \pm 0.008$ & 2 \\
$V-R_{\mathrm{C}}$ & & $0.526 \pm 0.007$ & 2 \\
$V-I_{\mathrm{C}}$ & & $0.994 \pm 0.007$ & 2 \\
$J$ & & $12.111 \pm 0.027$ & 3 \\
$J-H$ & & $0.438 \pm 0.035$ & 3 \\
$J-K_{s}$ & & $0.547 \pm 0.033$ & 3 \\
$M_{\star}$ & $M_{\odot}$ & $0.893 \pm 0.024$ & 2 \\
$R_{\star}$ & $R_{\odot}$ & $0.866 \pm 0.013$ & 2 \\
$T_{\text {eff }}$ & $\mathrm{K}$ & $5171 \pm 36$ & 2 \\
{$[$ Fe $/ \mathrm{H}]$} & & $+0.20 \pm 0.08$ & 2 \\
$\log g$ & & $4.513 \pm 0.013$ & 2 \\
$v$ sin $i$ & $\mathrm{~km} \mathrm{~s}$ & $3.80 \pm 0.36$ & 2 \\
$M_{V}$ & & $+5.756 \pm 0.041$ & 2 \\
Distance & $\mathrm{pc}$ & $360 \pm 11$ & 2 \\
Age & $\mathrm{Gy}$ & $7.38 \pm 1.87$ & 2 \\
\hline
\end{tabular}

References. (1) UCAC3 (Zacharias et al. 2010);

(2) this paper; (3) 2MASS (Skrutskie et al. 2006).

Table 2

Radial Velocity Measurements of TrES-5

\begin{tabular}{lr}
\hline \hline HJD & \multicolumn{1}{c}{ RV $\left(\mathrm{m} \mathrm{s}^{-1}\right)$} \\
\hline 2455456.847578 & $-542.8 \pm 27.9$ \\
2455468.707361 & $-472.6 \pm 23.3$ \\
2455647.005138 & $-308.3 \pm 22.8$ \\
2455649.002656 & $127.2 \pm 26.9$ \\
2455651.985839 & $98.3 \pm 29.0$ \\
2455659.987762 & $-549.8 \pm 29.9$ \\
2455667.970142 & $0.0 \pm 14.8$ \\
2455673.945954 & $45.0 \pm 14.8$ \\
\hline
\end{tabular}

and cross-correlation, see Buchhave et al. (2010), in which the details of the same reduction package are described, as applied to the Fiber-fed Echelle Spectrograph. The absolute gamma velocity, calculated using the weighted mean offset of the relative velocities from the corresponding absolute velocities, was determined to be $\gamma=-13.403 \pm 0.100 \mathrm{~km} \mathrm{~s}^{-1}$. The absolute gamma velocity is onto the system in which HD 182488 is defined to have an absolute velocity of $-21.508 \mathrm{~km} \mathrm{~s}^{-1}$.

To derive the stellar atmospheric parameters, the observed spectra were cross-correlated against a grid of synthetic spectra drawn from the library of spectra described above. The synthetic spectra cover a window of $300 \AA$ centered near the gravitysensitive $\mathrm{Mg} \mathrm{b}$ triplet and have a spacing of $250 \mathrm{~K}$ in effective temperature, $0.5 \mathrm{dex}$ in gravity, $0.5 \mathrm{dex}$ in metallicity, and $1 \mathrm{~km} \mathrm{~s}^{-1}$ in rotational velocity. The best matched template to the observed spectrum represents the best matched stellar parameters on the library grid. A new set of tools is then used to derive more precise stellar parameters from the normalized cross-correlation peaks. A description of the tools will be published in L. A. Buchhave et al. (2011, in preparation).

We used the multicolor Johnson-Cousins photometry to form a variety of color indices and used the color-temperature calibrations by Ramírez \& Meléndez (2005) and Casagrande et al. (2006) to estimate the star's effective temperature $T_{\text {eff }}$. The average result, $T_{\text {eff }}=4943 \pm 36 \mathrm{~K}$, is $228 \mathrm{~K}$ cooler than 


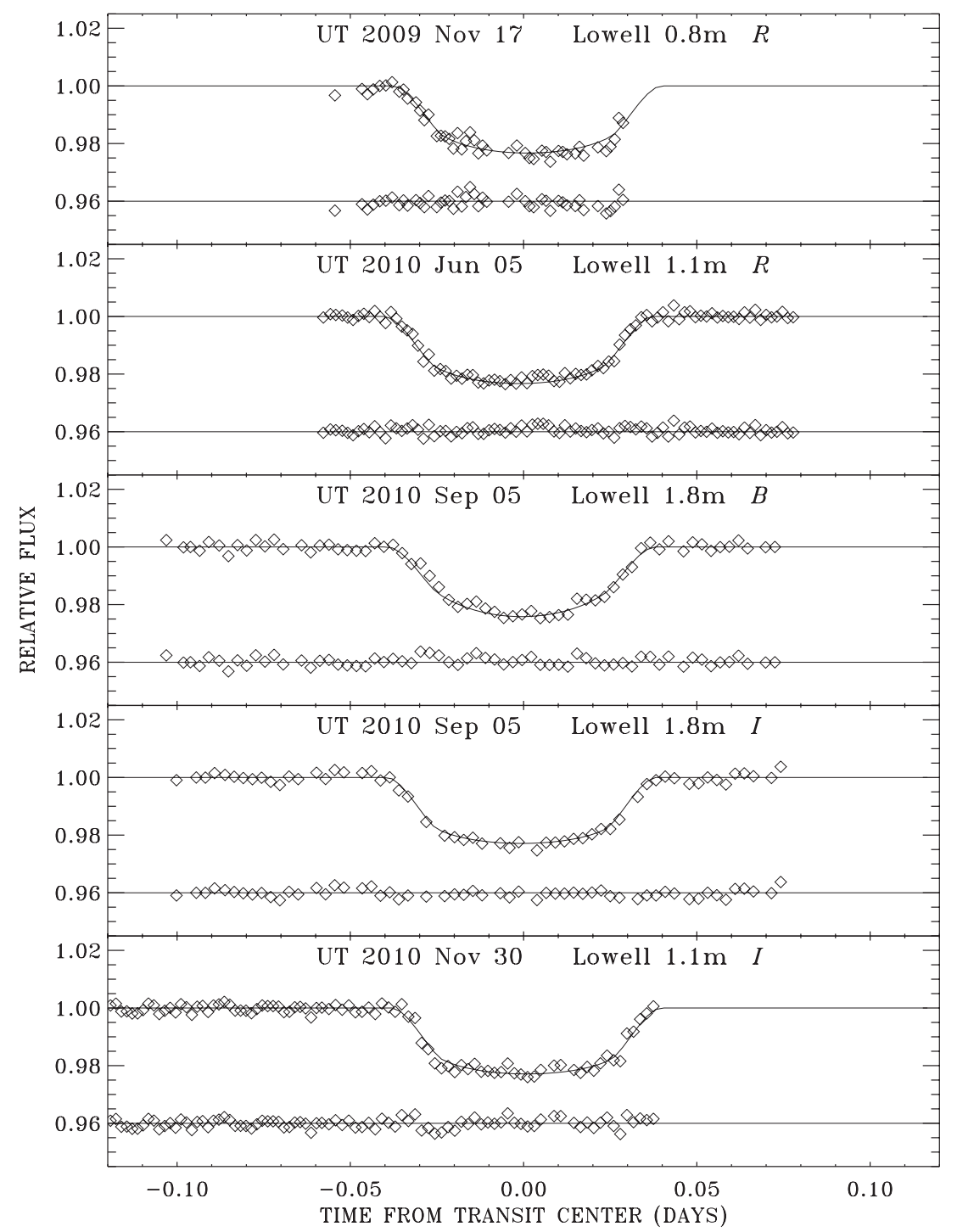

Figure 2. High-precision followup $B-, V$-, and $R$-band photometry of TrES-5. The plot shows the relative flux of the TrES-5 system as a function of time relative to the center of transit, adopting the ephemeris in Table 3. Each light curve is labeled with the telescope and date of observation. The residuals from the simultaneous fits (overplotted with solid lines) are shown below each light curve.

the spectroscopic value of $T_{\text {eff }}=5171 \pm 36 \mathrm{~K}$ (see below). This result is not surprising as some reddening is expected at the object's Galactic latitude of $13^{\circ}$. From the match to stellar evolution models described below, we estimate a reddening of $E_{B-V} \approx 0.07$, which is in good agreement with the difference of $228 \mathrm{~K}$ between the spectroscopic and photometric effective temperatures.

\section{LIGHT CURVE ANALYSIS}

We analyzed the five $B$-, $R$-, and $I$-band transit observations using the analytical expressions in Mandel \& Agol (2002) to compute the model flux. We assumed a circular orbit and a quadratic stellar limb-darkening law, fixing the coefficients at the color-dependent values tabulated in Claret (2004) for the spectroscopically estimated $T_{\text {eff }}, \log g$, and $[\mathrm{Fe} / \mathrm{H}]$. Initially, we adopted as free parameters the orbital period $P$ and epoch $T_{0}$, the ratio of the planet radius to the stellar radius $R_{\mathrm{p}} / R_{\star}$, the square of the impact parameter $b=\left(a / R_{\star}\right) \cos i$, where $a$ is the semimajor axis of the planet's orbit and $i$ is the orbital inclination, the parameter $\zeta / R_{\star}$, which for zero eccentricity is related to $a / R_{\star}$ via $\zeta / R_{\star}=\left(a / R_{\star}\right)(2 \pi / P)\left(1-b^{2}\right)^{-1 / 2}$, and the zero point of the out-of-transit flux $F_{0}$. This choice of parameters minimizes the correlation between $b$ and $a / R_{\star}$ (see Bakos et al. 2007). We performed an initial fit using the high-precision follow-up transit photometry, after which we fixed all parameters except $P$ and $T_{0}$ to their values from the initial fit, and repeated the fit using both the PSST wide-field photometry and the follow-up photometry. This approach allowed us to utilize the entire baseline of over three years of observations and obtain more precise values for $P$ and $T_{0}$ than if only the followup photometry were used. We then fixed the values of $T_{0}$ and $P$ (stated in Table 3 ) in the subsequent analysis.

We performed a joint fit of all five transit light curves using the MPFIT package (Markwardt 2008) and found the values of $R_{\mathrm{p}} / R_{\star}, b^{2}, \zeta / R_{\star}$, and $F_{0}$ that minimized the $\chi^{2}$. This fit is shown with solid lines in Figure 2. In order to estimate the errors of the fitted parameters, we conducted a Markov Chain Monte Carlo (MCMC) analysis (see Ford 2005 and references therein). Altogether 10 independent Markov chains with $5 \times 10^{5}$ points per chain were created, each chain starting 
Table 3

TrES-5 Planet Parameters

\begin{tabular}{llc}
\hline \hline Parameter & Units & Value \\
\hline$P$ & days & $1.4822446 \pm 0.0000007$ \\
$T_{0}$ & HJD & $2455443.25153 \pm 0.0001069$ \\
$a$ & AU & $0.02446 \pm 0.00068$ \\
$i$ & deg & $84.529 \pm 0.005$ \\
$a / R_{\star}$ & & $6.074 \pm 0.143$ \\
$b=a \cos i / R_{\star}$ & & $0.579 \pm 0.026$ \\
$K$ & $\mathrm{~m} \mathrm{~s}^{-1}$ & $339.8 \pm 10.4$ \\
$M_{\mathrm{p}}$ & $M_{\mathrm{J}}$ & $1.778 \pm 0.063$ \\
$R_{\mathrm{p}}$ & $R_{\mathrm{J}}$ & $1.209 \pm 0.021$ \\
$\bar{\rho}$ & $\mathrm{g} \mathrm{cm}$ & $1.25 \pm 0.08$ \\
$T_{\mathrm{eq}}$ & $\mathrm{K}$ & $1484 \pm 41$ \\
$R_{\mathrm{p}} / R_{\star}$ & & $0.1436 \pm 0.0012$ \\
\hline
\end{tabular}

Note. $M_{\mathrm{J}}=1.899 \times 10^{27} \mathrm{~kg}$ (Jupiter's mass); $R_{\mathrm{J}}=7.1492 \times 10^{7} \mathrm{~m}$ (Jupiter's equatorial radius; Cox 2000).

from a random perturbation of the best-fit parameters. We discarded the first $20 \%$ of the points to minimize the impact of the initial conditions. The resulting histograms of $4 \times 10^{6}$ values were used to obtain the mode and the $68.3 \%$ lower and upper confidence limits $p_{\text {lo }}$ and $p_{\text {up }}$ for each parameter. We then adopted the mode as the final, optimal value of the parameter, and the value of $\left(p_{\text {up }}-p_{\text {lo }}\right) / 2$ as its $1 \sigma$ error (encompassing $68.3 \%$ of the parameter values around the mode). The use of a single number to characterize the width of each parameter's distribution is justified by the nearly Gaussian, symmetric shape of all histograms.

\section{PROPERTIES OF TrES-5 AND ITS STAR}

The mass and radius of the host star of TrES-5, required for establishing the planet properties, were determined on the basis of the spectroscopic $T_{\text {eff }}$ and $[\mathrm{Fe} / \mathrm{H}]$, and the value of $a / R_{\star}$ derived from the light curve fit described above. The quantity $a / R_{\star}$ is closely related to the stellar density and is determined in this case with higher relative precision than $\log g$. It is therefore a better proxy for luminosity (see Sozzetti et al. 2007).

We used an iterative procedure to determine the stars' parameters, similar to the one described in Torres et al. (2008). Using the stellar evolution models from the series by Yi et al. (2001), we computed isochrones over a range in stellar age, from $0.1 \mathrm{~Gy}$ to $12 \mathrm{~Gy}$, over the metallicity range allowed by the spectroscopy. We compared the measured $T_{\text {eff }}$ and $a / R_{\star}$ from the light curve fit to the computed values of $a / R_{\star}$ and $T_{\text {eff }}$ along each isochrone and recorded the points on the isochrone that matched the measured quantities within their errors. We then calculated the weighted mean of all matches, with weights inversely proportional to the exponent of the distance (in $\chi^{2}$ sense) between the observed and model values of $T_{\text {eff }}, a / R_{\star}$, and $[\mathrm{Fe} / \mathrm{H}]$ (see Torres et al. 2008).

This procedure yields a value of $\log g$ which is better constrained than the spectroscopic estimate. With the value of $\log g$ fixed, revised estimates of $T_{\text {eff }}$ and $[\mathrm{Fe} / \mathrm{H}]$ were derived from the spectra, and the light curve fit repeated. The new values of $T_{\text {eff }}$ and $[\mathrm{Fe} / \mathrm{H}]$ (from spectroscopy), and $a / R_{\star}$ (from the fit) were again compared to the stellar models to obtain the final values of $M_{\star}=0.893 \pm 0.024 M_{\odot}, R_{\star}=0.866 \pm 0.013 R_{\odot}$, $\log g=4.513 \pm 0.013$, and age of $7.38 \pm 1.87 \mathrm{~Gy}$.

We fit a Keplerian orbit to these data assuming zero eccentricity as a good first approximation, as expected from theoretical arguments for a period as short as 1.48 days. The period and

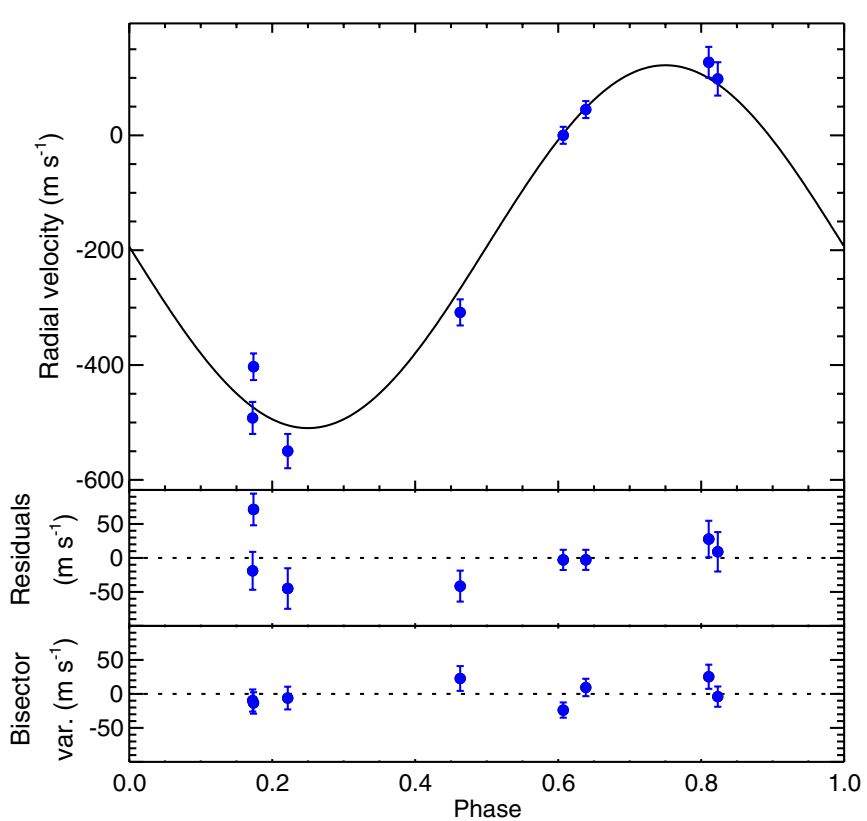

Figure 3. Top: radial velocity observations of TrES-5 obtained with TRES, shown relative to the center of mass and adopting the ephemeris in Table 3 . The best-fit orbit (solid line) is overplotted. Middle: residuals from the best-fit model to the radial velocities. Bottom: bisector spans shifted to a median of zero, for each of the TRES exposures.

(A color version of this figure is available in the online journal.)

epoch were held fixed. The rms of this fit is $24.2 \mathrm{~m} \mathrm{~s}^{-1}$, which is similar to the internal errors of the velocities. The parameters of this orbital solution are listed in Table 3. The orbit is displayed in Figure 3 (top panel) along with the observations, and the residuals are shown in the middle panel.

We investigated the possibility that the radial velocities we measured are the result of distortions in the line profiles due to contamination from an unresolved eclipsing binary (Santos et al. 2002; Torres et al. 2005) or starspots (Toner \& Gray 1988), instead of being due to true Doppler motion in response to a planetary companion. We cross-correlated each TRES spectrum against a synthetic template matching the properties of the star and averaged the correlation functions over the orders. From this representation of the average spectral line profile we computed the mean bisectors, and as a measure of the line asymmetry we calculated the "bisector spans" as the velocity difference between points selected near the top and bottom of the mean bisectors (Toner \& Gray 1988; Torres et al. 2005). If the velocities were the result of a blend with an eclipsing binary (Mandushev et al. 2005) or starspots (Queloz et al. 2001), we would expect the line bisectors to vary in phase with the photometric period with an amplitude similar to that of the velocities. Instead, we detect no variation in excess of the measurement uncertainties (see Figure 3, bottom panel), and we conclude that the velocity variations are real and that the star is orbited by a Jovian planet.

\section{DISCUSSION}

TrES-5 orbits one of the faintest host stars with transiting planets found to date. The discovery demonstrates that precise photometry and spectroscopy are possible from the ground even for such faint stars, although some follow-up studies will be more challenging. With a period of only 1.5 days, TrES-5 is a classical "hot Jupiter," although the relatively cool host star 
$\left(T_{\text {eff }}=5171 \mathrm{~K}\right)$ places it near the upper (hotter) end of the transition zone between the "pM class" and "pL class" planets proposed in Fortney et al. (2008). In such planets the importance of gaseous $\mathrm{TiO}$ and $\mathrm{VO}$ absorption is diminished, temperature inversion may not be present, and the flux from the host star is more evenly distributed around the planet and inside its interior. Because of its location near the $\mathrm{pM} / \mathrm{pL}$ boundary, TrES-5 could be a suitable object for testing the models of planetary atmospheres.

At $M_{\mathrm{p}}=1.8 M_{\mathrm{J}} \mathrm{TrES}-5$ is above the upper quartile of the planetary mass distribution. The mass and radius of TrES-5 are in good agreement with the theoretical mass-radius relations for irradiated planets (Fortney et al. 2007; Hansen \& Barman 2007). For an age of $7.5 \mathrm{Gyr}$, planetary mass of $M_{\mathrm{p}}=1.8 M_{\mathrm{J}}$, and equilibrium temperature of $T_{\mathrm{eq}} \sim 1500 \mathrm{~K}$, the predicted radius of the planet is $R_{\mathrm{p}} \sim 1.2 R_{\mathrm{J}}$, which is in good agreement with the observed value. Thus, TrES-5 does not appear to have the anomalously large radius of many close-in giant exoplanets. A comparison with the planetary models of Fortney et al. (2007) for the mass, age, and semimajor axis of TrES-5 indicates that the planet is best approximated by a model with no heavyelements core.

It has been noted that many massive planets have non-zero eccentricity even at short periods (Southworth et al. 2009). Using the current catalog of transiting planets at http://exoplanet.eu, we find that over a third of the short-period, massive planets $\left(P<3\right.$ days and $\left.M_{\mathrm{p}}>1.7 M_{\mathrm{J}}\right)$ have non-zero eccentricities. In our radial-velocity fit we have assumed $e=0$ based on the short period of TrES-5 and theoretical arguments about the timescales of tidal interactions (Zahn \& Bouchet 1989). Our radial velocities clearly reject large eccentricities. From MCMC analysis of the radial-velocity data, we find that the preferred orbital solution has $e=0.025_{-0.025}^{+0.015}$. We therefore adopt $e=$ 0 for the orbit of TrES-5, but we cannot rule out a small eccentricity $(e<0.04)$. A future detection of the secondary eclipse will help constrain the orbital eccentricity much better, as well as test for the presence of temperature inversion in the atmosphere of TrES-5.

We thank Travis Barman for useful discussions. This paper is based on work supported in part by NASA grants NNG04GN74G, NNG04LG89G, NNG05GI57G, NNG05GJ29G, and NNH05AB88I through the Origins of Solar Systems Program, and NASA Planetary Major Equipment grant N4G5-12229. We acknowledge support from the NASA Kepler mission under cooperative agreement NCC2-1390, and M.R. acknowledges support from ALMA-CONICYT projects
31090015 and 31080021. This publication makes use of data products from the Two Micron All Sky Survey, which is a joint project of the University of Massachusetts and the Infrared Processing and Analysis Center/California Institute of Technology, funded by NASA and the National Science Foundation.

\section{REFERENCES}

Baglin, A., Auvergne, M., Barge, P., et al. 2009, in IAU Symp. 253, Transiting Planets, ed. F. Pont, D. Sasselov, \& M. Holman (Cambridge: Cambridge Univ. Press), 71

Bakos, G. Á., Shporer, A., Pál, A., et al. 2007, ApJ, 671, L173

Baraffe, I., Chabrier, G., \& Barman, T. 2010, Rep. Prog. Phys., 73, 016901

Borucki, W. J., Koch, D., Basri, G., et al. 2010, Science, 327, 977

Buchhave, L. A., Bakos, G. Á., Hartman, J. D., et al. 2010, ApJ, 720, 1118

Casagrande, L., Portinari, L., \& Flynn, C. 2006, MNRAS, 373, 13

Charbonneau, D., Brown, T. M., Burrows, A., \& Laughlin, G. 2007, in Protostars and Planets V, ed. B. Reipurth, D. Jewitt, \& K. Keil (Tucson, AZ: Univ. Arizona Press), 701

Chatterjee, S., Ford, E. B., Matsumura, S., \& Rasio, F. A. 2008, ApJ, 686, 580

Claret, A. 2004, A\&A, 428, 1001

Cox, A. N. (ed.) 2000, Allen's Astrophysical Quantities (4th ed.; New York: AIP Press), 295

Dunham, E. W., Mandushev, G., Taylor, B., \& Oetiker, B. 2004, PASP, 116 , 1072

Ford, E. B. 2005, AJ, 129, 1706

Fortney, J. J., Lodders, K., Marley, M. S., \& Freedman, R. S. 2008, ApJ, 678, 1419

Fortney, J. J., Marley, M. S., \& Barnes, J. W. 2007, ApJ, 659, 1661

Fürész, G. 2008, PhD thesis, Univ. Szeged, Hungary

Hansen, B. M. S., \& Barman, T. 2007, ApJ, 671, 861

Landolt, A. U. 1992, AJ, 104, 340

Latham, D. W., Stefanik, R. P., Mazeh, T., Mayor, M., \& Burki, G. 1989, Nature, 339, 38

Latham, D. W., Stefanik, R. P., Torres, G., et al. 2002, AJ, 124, 1144

Mandel, K., \& Agol, E. 2002, ApJ, 580, L171

Mandushev, G., Torres, G., Latham, D. W., et al. 2005, ApJ, 621, 1061

Markwardt, C. B. 2008, in ASP Conf. Ser. 411, Astronomical Data Analysis Software and Systems XVIII, ed. D. Bohlender, P. Dowler, \& D. Durand (San Francisco, CA: ASP), 251

Mayor, M., \& Queloz, D. 1995, Nature, 378, 355

Nordström, B., Latham, D. W., Morse, J. A., et al. 1994, A\&A, 287, 338

Queloz, D., Henry, G. W., Sivan, J. P., et al. 2001, A\&A, 379, 279

Ramírez, I., \& Meléndez, J. 2005, ApJ, 626, 465

Santos, N. C., Mayor, M., Naef, D., et al. 2002, A\&A, 392, 215

Skrutskie, M. F., Cutri, R. M., Stiening, R., et al. 2006, AJ, 131, 1163

Southworth, J., Hinse, T. C., Dominik, M., et al. 2009, ApJ, 707, 167

Sozzetti, A., Torres, G., Charbonneau, D., et al. 2007, ApJ, 664, 1190

Struve, O. 1952, The Observatory, 72, 199

Toner, G. G., \& Gray, D. F. 1988, ApJ, 334, 1008

Torres, G., Konacki, M., Sasselov, D. D., \& Jha, S. 2005, ApJ, 619, 558

Torres, G., Winn, J. N., \& Holman, M. J. 2008, ApJ, 677, 1324

Yi, S. K., Demarque, P., Kim, Y.-C., et al. 2001, ApJS, 136, 417

Zacharias, N., Finch, C., Girard, T., et al. 2010, AJ, 139, 2184

Zahn, J.-P., \& Bouchet, L. 1989, A\&A, 223, 112 